\title{
Spiral-Scan Atomic Force Microscopy: A Constant Linear Velocity Approach
}

\author{
Iskandar A. Mahmood and S. O. Reza Moheimani, Senior Member, IEEE
}

\begin{abstract}
This paper describes an alternative method to the widely-used raster-scan technique for Atomic Force Microscopy (AFM). In this method, the sample is scanned in a spiral pattern instead of the well established raster trajectory. A spiral pattern is produced by applying cosine and sine signals with slowly varying amplitudes to the $x$-axis and $y$-axis of an AFM scanner respectively. In order to ensure that the spiral trajectory travels at a constant linear velocity (CLV), frequency and amplitude of the input signals are varied simultaneously in a way that the linear velocity of the scanner is kept constant. Experimental results obtained by implementing the CLV spiral scan on a commercial AFM indicate that, compared with the raster-scan method, highquality AFM images of equal area and pitch can be generated two times faster and using half of the total traveled distance.
\end{abstract}

\section{INTRODUCTION}

Since its invention, Atomic Force Microscope (AFM) [1], [2] has become an important research instrument in various applications of nanoscience and nanotechnology. The basic components of AFM, as illustrated in Fig. 1, include a microcantilever with a sharp tip at its free end, a laser-photodetector sensor and a scanner. During operation, a sample is placed on the scanner and the tip of the micro-cantilever is brought very close to the sample surface at a distance of the order of a few nanometers. At such a distance, depending on AFM operating mode, the interactive forces change static or dynamic properties of the micro-cantilever. The changes in the microcantilever are often measured using a laser-photodetector sensor. These measurements can be used directly to generate the sample surface topography or as a feedback signal to the $z$ axis controller to keep the interactive forces nearly constant. In general, AFM images are typically generated by scanning the sample in a raster pattern.

In [3], the authors showed how a spiral trajectory, see Fig. 2, that travels at a constant angular velocity (CAV) is produced by applying slowly varying-amplitude single frequency sinusoidal signals to the $x$ and $y$ axes of the piezoelectric tube scanner. The use of the single frequency input signals allows for scanning to be performed at very high speeds well beyond what is practically achievable with the raster scans. However, a drawback of this method is that the linear velocity of the spiral trajectory is not constant. Thus, it may not be suitable for scanning samples where the interaction between the probe and the sample needs to be done at linear velocity. Here, we present a method to generate a spiral scan that travels at a constant linear velocity (CLV) to overcome this drawback.

I. A. Mahmood and S. O. R. Moheimani are with School of Electrical Engineering and Computer Science, The University of Newcastle, Callaghan, NSW 2308, Australia. Email: Reza.Moheimani@newcastle.edu.au.

I. A. Mahmood is on leave from Department of Mechatronics, Faculty of Engineering, International Islamic University Malaysia, 50728, Kuala Lumpur, Malaysia.

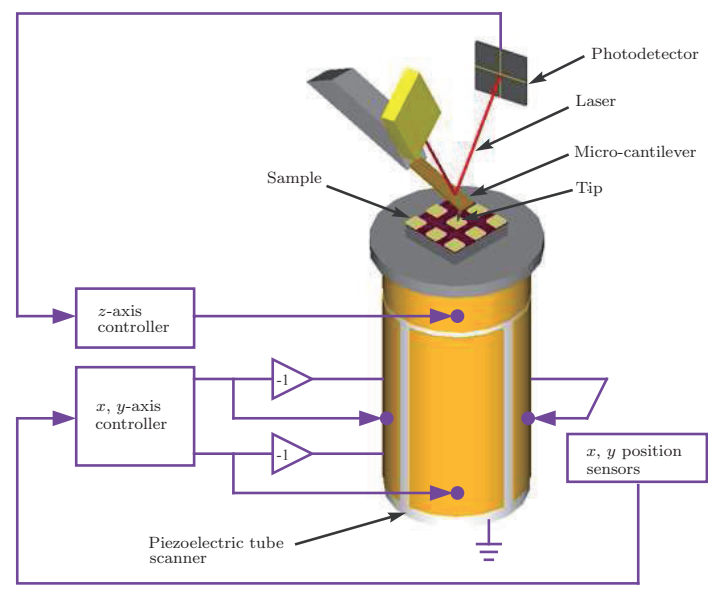

Figure 1. Basic AFM schematic with feedback controllers.

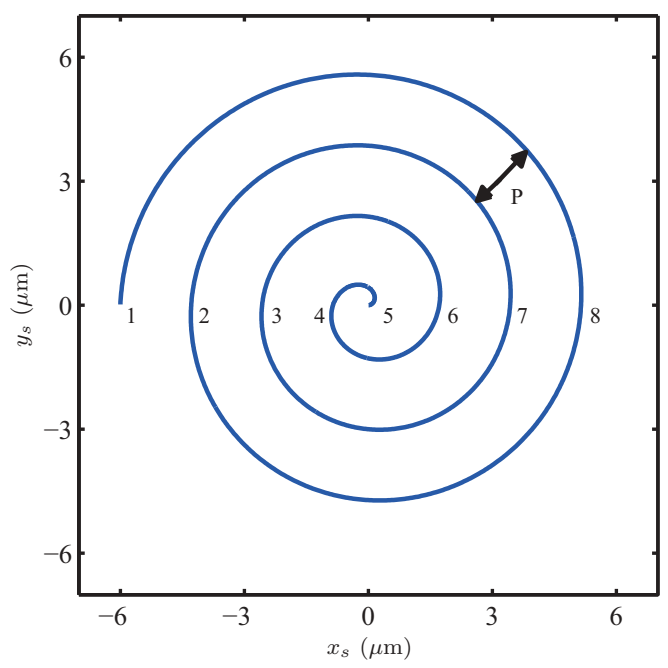

Figure 2. Spiral scan of $6 \mu \mathrm{m}$ radius with number of curve $=8$.

This scanning method has been implemented in some disk storage devices, such as Compact Disk-Read Only Memory (CD-ROM) where the information is stored in a continuous spiral track over the disk's surface [4].

The remainder of this paper is set out as follows. Section II describes in detail the generation of input signals to produce the CLV spiral pattern. Section III provides descriptions of the experimental setups and system identification approach undertaken in this work. Control schemes for tracking of the CLV spiral are devised in Section IV. In Section V experimental results are presented. Finally, section VI concludes the paper. 


\section{CLV SPIRAL SCAN}

\section{A. Input signals generation}

In order to generate a CLV spiral, the radius $r$ and angular velocity $\omega$ need to be varied simultaneously in a way that the linear velocity $v$ of the nanopositioner is kept constant at all times. The expressions for $r$ and $\omega$ can be derived by substituting $\omega=\frac{v}{r}$ into a differential equation given in [4] as

to obtain

$$
\frac{d r}{d t}=\frac{P \omega}{2 \pi}
$$

$$
\frac{d r}{d t}=\frac{P v}{2 \pi r}
$$

where $r$ is the instantaneous radius at time $t$ and $P$ is the pitch of the CLV spiral. Then, equation (2) is solved for $r$ by integrating both sides of the equation as

$$
\int r d r=\frac{P v}{2 \pi} \int d t
$$

For $r=0$ at $t=0$, we obtain

$$
r=\sqrt{\frac{P v}{\pi} t}
$$

From equation (4), by substituting $r=\frac{v}{\omega}$, the expression for $\omega$ is obtained as

$$
\omega=\sqrt{\frac{\pi v}{P t}} .
$$

The equation for total scanning time $T_{\text {spiral }}$ of a CLV spiral scan can be derived by integrating both sides of equation (2) as

$$
\int_{r_{\text {start }}}^{r_{\text {end }}} r d r=\frac{P v}{2 \pi} \int_{t_{\text {start }}}^{t_{\text {end }}} d t
$$

where $r_{\text {start }}$ and $r_{\text {end }}$ are initial and final values of the spiral radius, and $t_{\text {start }}$ and $t_{\text {end }}$ are initial and final values of the scanning time. From equation (6), if $r_{\text {start }}=0$ at $t_{\text {start }}=0$ and $T_{\text {spiral }}=t_{\text {end }}-t_{\text {start }}$, we obtain

$$
T_{\text {spiral }}=\frac{\pi r_{\text {end }}^{2}}{P v} .
$$

From equation (7), the total trajectory distance of a spiral scan $S_{\text {total }}$ can be derived as

$$
S_{\text {spiral }}=\frac{\pi r_{\text {end }}^{2}}{P} .
$$

In order to implement the spiral scans using a piezoelectric tube scanner, equation (4) is described in cartesian coordinates as

$$
x_{s}=r \cos \theta
$$

and

$$
y_{s}=r \sin \theta
$$

where $\theta$ for time varying $\omega$ is obtained as

$$
\theta=\sqrt{\frac{4 \pi v}{P} t}
$$

In this work, the input signals are implemented in a reversed order, that is from $r_{\text {end }}$ to $r_{\text {start }}$. To generate a CLV spiral, that starts from $r=0$, one requires a closed-loop system with extremely high bandwidth (ideally $\infty$ bandwidth) and a closed loop system with a flat phase and magnitude response. This of course, is not practical. Thus, if the spiral is started from $r=0$, the initial error that is inevitably generated will propagate all the way through to the end.

\section{B. Inversion Technique for CLV spiral}

In this section a technique to shape inputs such that the resulting trajectory will be a CLV spiral with minimal tracking error is presented. As the implementation of the entire scheme will be in discrete time, the input shaping method presented here is also described in discrete time.

The goal is to design input signals $\left\{u_{x}[k]\right\}_{k=0}^{N}$ and $\left\{u_{y}[k]\right\}_{k=0}^{N}$ such that their outputs, along the $x$ and $y$ axes are, $\{x[k]=\tilde{x}(k T)\}_{k=0}^{N}$ and $\{y[k]=\tilde{y}(k T)\}_{k=0}^{N}$ respectively. Here, $T$ denotes the sampling interval and $\tilde{x}$ and $\tilde{y}$ are as defined in equations (9) and (10). In the following only designing of $\left\{u_{x}[k]\right\}_{k=0}^{N}$ will be described, with the understanding that $\left\{u_{y}[k]\right\}_{k=0}^{N}$ can be generated by adopting the same procedure.

Assume that the transfer function relating the input and the output along the $x$ direction is given by

$$
G_{x}(z)=\frac{b_{0}+b_{1} z^{-1}+b_{2} z^{-2}+\ldots+b_{m} z^{-m}}{1+a_{1} z^{-1}+a_{2} z^{-2}+\ldots+a_{m} z^{-m}},
$$

which is stable but has non-minimum phase zeros, i.e. all of zeros are outside the unit circle. As $G_{x}(z)$ is non-minimum phase, a direct inversion is not possible. Furthermore, as $\tilde{x}$ and $\tilde{y}$ are not periodic, a frequency domain inversion of the type presented in [5] will not be accurate.

Note that equation (12) in the discrete time corresponds to the difference equation

$$
\begin{aligned}
& x[n]+a_{1} x[n-1]+\ldots+a_{m} x[n-m] \\
& =b_{0} u_{x}[n]+b_{1} u_{x}[n-1]+\ldots+b_{m} u_{x}[n-m] .
\end{aligned}
$$

This implies

$$
\begin{aligned}
& u_{x}[n-m]=\frac{1}{b_{m}}\left(x[n]+a_{1} x[n-1]+\ldots+a_{m} x[n-m]\right. \\
& \left.-b_{n} u_{x}[n]-\ldots-b_{m-1} u_{x}[n-(m-1)]\right) .
\end{aligned}
$$

As $\{x[k]\}_{k=0}^{N}$ is given, assuming arbitrary values for $u_{x}[N], u_{x}[N-1], \ldots, u_{x}[N-(m-1)]$, the input sequence $u_{x}[N-$ $(m-1)], u_{x}[N-(m-2)], \ldots, u_{x}[1], u_{x}[0]$ can be calculated from equation (14). As an example consider $m=2$ in (13). This implies

$$
\begin{aligned}
& x[n]+a_{1} x[n-1]+a_{2} x[n-2] \\
& =b_{0} u_{x}[n]+b_{1} u_{x}[n-1]+b_{2} u_{x}[n-2]
\end{aligned}
$$

and

$$
\begin{aligned}
& u_{x}[n-2]=\frac{1}{b_{2}}\left(x[n]+a_{1} x[n-1]\right. \\
& \left.+a_{2} x[n-2]-b_{0} u_{x}[n]-b_{1} u_{x}[n-1]\right) .
\end{aligned}
$$

Setting $u_{x}[N]$ and $u_{x}[N-1]$ to arbitrary values, $u_{x}[N-2]$ can be back calculated from equation (16). Similarly, using the calculated $u_{x}[N-2]$ and the arbitrarily chosen $u_{x}[N-1]$, $u_{x}[N-3]$ can be computed. Thus, traversing backwards in time one can compute $u_{x}[n]$ up to $n=0$. 
The above mentioned procedure can be proved to be stable, and can be shown to converge to an input sequence that would generate the output $\tilde{x}(k T)$. However, the proof is beyond the scope of this paper. If a user has to deal with a continuous time transfer function $G_{x}(s)$, he or she could approximate it by a discrete transfer function $G_{x}(z)$ using the bilinear transformation or any other approximation technique.

\section{Spiral scan vs. Raster scan}

A fair comparison of the total scanning time for spiral and raster scans can be made by evaluating the time required for both methods to generate images of equal areas and pitch lengths. The area of a circular spiral scanned image $A_{\text {spiral }}$ with a radius of $r_{\text {end }}$ can be calculated as

$$
A_{\text {spiral }}=\pi r_{\text {end }}^{2} \text {. }
$$

The area of a rectangular raster scanned image $A_{\text {raster }}$ can be calculated using

$$
A_{\text {raster }}=L^{2}
$$

where $L$ is length of the square image. For both images to have an equal area, equations (17) and (18) are equated to obtain

$$
L=\sqrt{\pi} r_{\text {end }} .
$$

The number of lines in a raster scanned image with pitch $P$ can be calculated as

$$
\text { number of lines }=\frac{L}{P}+1 \text {. }
$$

The total scan time to generate a raster scanned image can be obtained using

$$
T_{\text {raster }}=\frac{\text { number of lines }}{f}
$$

where $f$ is the scan frequency. Thus, by substituting equations (19) and (20) into equation (21), the total scan time for generating a raster scanned image with an area of $\pi r_{\text {end }}^{2}$ can be determined as

$$
T_{\text {raster }}=\frac{\sqrt{\pi} r_{\text {end }}}{P f}+\frac{1}{f} .
$$

In order to compare the total scanning time for a CLV spiral scan and a raster scan, the linear velocity of the raster scan $v_{r}=2 L f$ is introduced into equation (22) to obtained

$$
T_{\text {raster }}=\frac{2 \pi r_{\text {end }}^{2}}{P v_{r}}
$$

with the term $\frac{1}{f}$ ignored. It can be deduced from equations (23) and (7) that for the same linear velocity, $v_{r}=v$, an image of equal area and pitch can be generated two times faster using a CLV spiral scan than a raster scan.

The total trajectory of a raster scan $S_{\text {raster }}$ can be written as

$$
S_{\text {raster }}=2 L \times \text { number of lines. }
$$

By substituting equations (20) and (19) into equation (24), $S_{\text {raster }}$ can be obtained as

$$
S_{\text {raster }}=\frac{2 \pi r_{\text {end }}^{2}}{P}+2 \sqrt{\pi} r_{\text {end }} .
$$

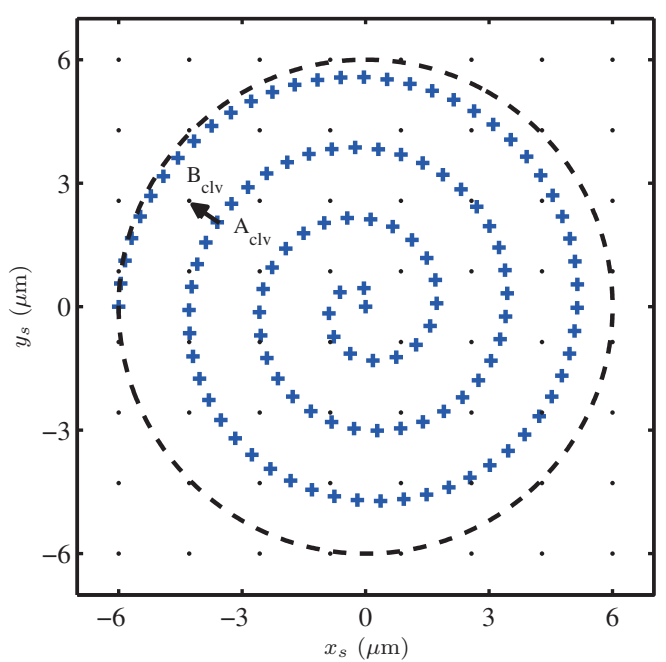

Figure 3. Spiral points (+) for CLV spiral with $v_{s}=1.1 \mathrm{~mm} / \mathrm{s}$. The sampling frequency used for generating these spiral points is $2 \mathrm{kHz}$. The spiral trajectories have a $6.5 \mu \mathrm{m}$ radius with number of curves $=8$. The spiral points are plotted on top of the raster points (.) that make up a $13 \times 13 \mu \mathrm{m}$ raster-scanned image with of $8 \times 8$ pixels resolution.

It can be deduced from equation (25) and (8) that, $S_{\text {raster }}$ is at least two times longer than $S_{\text {spiral }}$. An immediate advantage of this is that, there will be less wear on the AFM tip when the spiral scan is used instead of the raster scan.

\section{Mapping Spiral Points to Raster Points}

In this work, the spiral-scanned images are plotted by mapping the sampling points along the spiral trajectory (called "spiral points") to points or pixels (called "raster points") that make up a raster-scanned image placed on top of the spiral points as shown in Fig. 3. A major advantage of mapping the spiral points to the raster points is that it allows the user to utilize existing image processing software developed specifically for raster-scanned images, to plot the generated spiral image.

In this mapping procedure, the dimension of the rasterscanned image is set to spiral diameter $\times$ spiral diameter where the spiral diameter $\approx$ spiral radius $\times 2$, and the pitch of the raster-scanned image is chosen to be equal to the pitch $P$ of the spiral. Consequently, the number of lines in the raster-scanned image will be equal to the number of curves in the spiral trajectory. Then, each raster point located within the spiral radius is mapped to the nearest spiral point. Since position of the raster and spiral points are known for any scan frequency and dimension, the nearest spiral point corresponding to each raster point can be identified and stored in an indexed matrix before performing the sample scans. By doing this, the image of the sample can be plotted in real-time, i.e., as the AFM is scanning the sample.

Next, the error introduced by mapping the spiral points to the raster points is analyzed. This mapping error can be determined by calculating the magnitude of the vector between the nearest spiral point corresponding to each raster point. Fig. 3 illustrates an example of the vector between spiral point $A_{c l v}$ and raster point $B_{c l v}$ which corresponds to raster point 


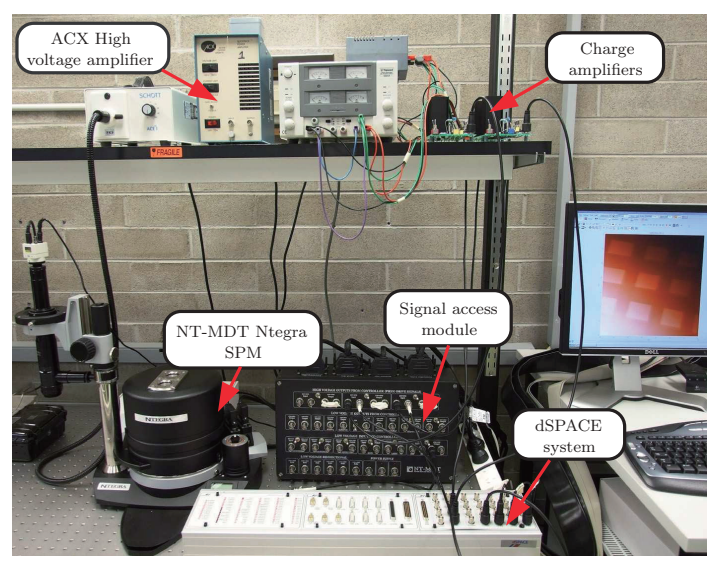

Figure 4. SPM system and experimental setup used in this work.

$(2,6)$. The magnitude of this vector can be calculated as

$$
\left|A B_{c l v}^{(i, j)}\right|=\sqrt{\left(A_{c l v}^{x}-B_{c l v}^{x}\right)^{2}+\left(A_{c l v}^{y}-B_{c l v}^{y}\right)^{2}}
$$

where $i=2$ and $j=6$. This mapping error can be ignored as long as it is less then the pitch of the spiral trajectory.

\section{EXPERIMENTAL SETUP AND SyStem IDENTIFICATION}

The experimental setup used in this work consisted of a commercial NT-MDT Ntegra Scanning Probe Microscope (SPM) as shown in Fig. 4. Here, the SPM was configured to operate as an AFM and all sample images were scanned in air. The voltage amplifiers which drive the lateral axes of the piezoelectric tube scanner were replaced with two home made DC-accurate charge amplifiers [6]. The use of the charge amplifiers to drive the piezoelectric tube has been shown to result in a reduction of the hysteresis by greater than $90 \%$ as compared with when voltage amplifiers are used [7]. A dSPACE-1103 rapid prototyping system was used to implement the feedback controllers in real time. The amplifiers and the SPM were interfaced with the dSPACE system using a signal access module (SAM) that allowed direct access to the scanner electrodes. This setup enabled us to directly control the lateral movements of the scanner. However, the scanner vertical positioning was achieved using the standard NT-MDT SPM controller. During scans, measurements from the capacitive sensors and photodiode were recorded and processed in Matlab to generate AFM images.

Next, models of the scanner were identified for the purpose of controller design. The scanner was treated as a two singleinput single-output (SISO) systems in parallel. The inputs being the voltage signals applied to the charge amplifiers driving $+x$ electrode pair, $u_{x}$ and $+y$ electrode pair, $u_{y}$. The outputs of the system are the scanner displacement measurements from the capacitive sensors in $x$ axis, $c_{x}$, and in $y$ axis, $c_{y}$. Here, accurate models of the systems were obtained through frequency domain subspace-based system identification approach described in [8]. A dual-channel HP35670A spectrum analyzer was used to obtain the following frequency response functions
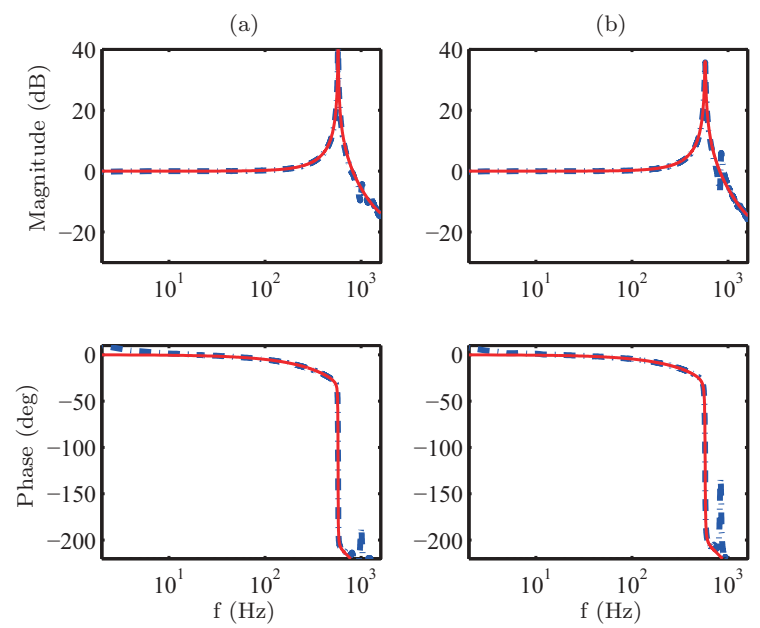

Figure 5. Experimental (--) and identified model (-) frequency response of (a) $G_{c_{x} u_{x}}(s)$ and (b) $G_{c_{y} u_{y}}(s)$.

(FRFs) nonparametrically

$$
G_{c_{x} u_{x}}(i \omega)=\frac{c_{x}(i \omega)}{u_{x}(i \omega)}
$$

and

$$
G_{c_{y} u_{y}}(i \omega)=\frac{c_{y}(i \omega)}{u_{y}(i \omega)} .
$$

A band-limited random noise signal of amplitude $0.5 \mathrm{Vpk}$ within the frequency range of $1 \mathrm{~Hz}$ to $1600 \mathrm{~Hz}$ was generated using the spectrum analyzer and applied to the charge amplifiers as the input. The corresponding outputs from the capacitive displacement sensors were also recorded using the spectrum analyzer. These input-output data were processed to generate the FRF (27) and (28) in a non-parametric form as illustrated in Fig. 5. Two second order models were fitted to the FRFs data using the frequency domain subspace-based system identification approach as described in [8] and [9]. The following transfer functions were found to be a good fit as illustrated in Fig. 5,

$$
G_{c_{x} u_{x}}(s)=\frac{0.1254 s^{2}-1784 s+1.309 \times 10^{7}}{s^{2}+28.35 s+1.309 \times 10^{7}}
$$

and

$$
G_{c_{y} u_{y}}(s)=\frac{0.1006 s^{2}-1610 s+1.318 \times 10^{7}}{s^{2}+57.59 s+1.318 \times 10^{7}} .
$$

\section{Controller Design}

It is worth mentioning that fairly accurate tracking of the spiral trajectory can be done in open-loop by shaping the input signals. However, in addition to this, closed-loop tracking was also undertaken in this work in order to damp the first resonant mode of the piezoelectric tube scanner and to minimize effects such as hysteresis, creep and drift [10]. Feedback controllers for the $x$ and $y$ axes were designed independently since the scanner is treated as a two SISO systems in parallel. Structure of the $x$-axis feedback controller is illustrated in Fig. 6. A similar controller was also designed for the $y$-axis. 


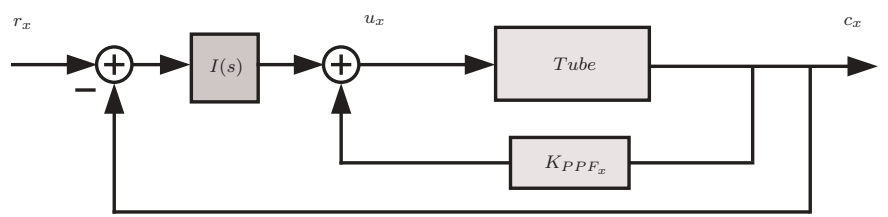

Figure 6. Structure of the $x$-axis feedback controller. The inner feedback loop is a positive position feedback (PPF) controller designed to damp the highly resonant mode of the tube. Integral action is also incorporated to achieve satisfactory tracking.

The overall control structure consists of an inner and an outer loop. The inner loop contains a second order Positive Position Feedback (PPF) [11] controller that works to increase the overall damping of the scanner. Their effectiveness in improving accuracy and bandwidth of nanopositioning systems was recently investigated in [5], and their important stability properties were established in [12]. PPF controllers have a number of important features. In particular, they have a simple structure which makes their implementation straight forward and their transfer functions roll off at a rate of $40 \mathrm{~dB} /$ decade at higher frequencies. The latter is important in terms of the overall effect of sensor noise on the scanner's positioning accuracy. The procedure that was followed to design these PPF controllers is well documented in reference [5]. The outer loop contains a high-gain integral controller to provide accurate tracking. The obtained PPF controllers can be described as

$$
K_{P P F_{x}}(s)=\frac{9.282 \times 10^{6}}{s^{2}+6062 s+2.736 \times 10^{7}}
$$

and

$$
K_{P P F_{y}}(s)=\frac{9.313 \times 10^{6}}{s^{2}+6071 s+2.758 \times 10^{7}} .
$$

In this work, the gain of the integrators were tuned to provide high closed-loop system bandwidth but with reasonable gain and phase margin.

\section{Results}

The closed-loop frequency responses of the piezoelectric tube scanner were first measured using the spectrum analyzer. Fig. 7 illustrates the closed-loop frequency responses when plotted together with the open-loop frequency responses of the system. By examining these FRFs we can see that a damping of more than $30 \mathrm{~dB}$ is apparent at each resonant mode. The use of the high-gain integral controllers has resulted in a high closed-loop system bandwidth of about $540 \mathrm{~Hz}$ in both axes. However, in the closed-loop system, the frequency responses exhibit a faster phase drop rate as compared to the openloop system. Consequently this results in greater phase shifts between the desired and the achieved trajectories. In this work, the phase shifts are handled by shaping the input signals as discussed in Section II-B.

The performance of the closed-loop systems were then evaluated for tracking of the CLV spirals. The experiment was setup to produce spiral scans with $r_{\text {end }}=6 \mu \mathrm{m}$ and number of curves $=512$, i.e. the diameter of the resulting circular image consists of 512 pixels. Fig. 8 (a), (b) and (c) illustrate the tracking trajectories between $\pm 0.30 \mu \mathrm{m}$ of
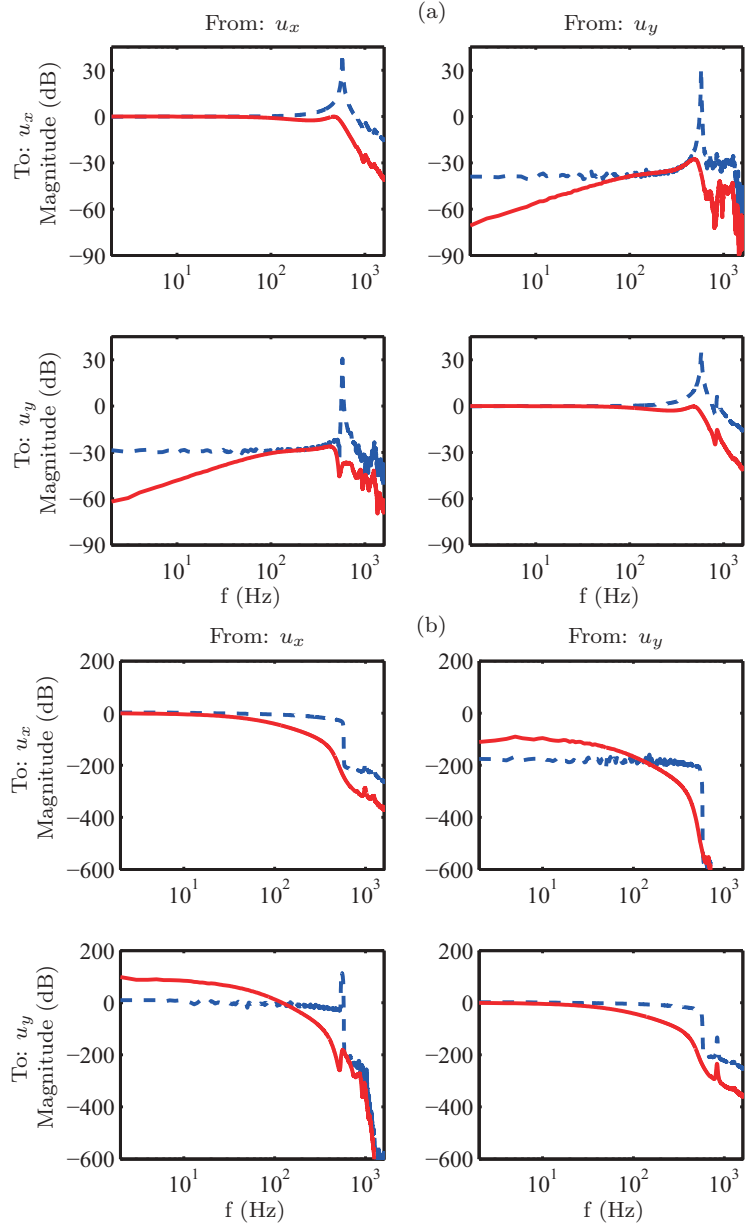

Figure 7. Open-loop (--) and closed-loop (-) frequency responses of the scanner.

Table I

RMS VALUES OF TRACKING ERROR, TOTAL SCANNING TIME AND SPIRAL TO RASTER POINTS MAPPING ERROR FOR CLV SPIRAL SCANS.

\begin{tabular}{|c|c|c|c|c|}
\hline$v_{s}(\mathrm{~mm} / \mathrm{s})$ & $f_{\text {samp }}(\mathrm{kHz})$ & $t_{\text {total }}(\mathrm{s})$ & $E_{\text {rms }}(\mathrm{nm})$ & $E_{\text {map }_{\text {RMS }}}(\mathrm{nm})$ \\
\hline 0.2 & 10 & 25.55 & 3.60 & 2.61 \\
0.6 & 20 & 8.52 & 7.26 & 2.83 \\
1.1 & 20 & 4.26 & 10.67 & 3.49 \\
\hline
\end{tabular}

the CLV spirals for $v_{s}=0.2,0.6$ and $1.1 \mathrm{~mm} / \mathrm{s}$. Fig. 8 (a) shows that relatively good tracking was obtained for $v_{s}=$ $0.2 \mathrm{~mm} / \mathrm{s}$. However, for $v_{s}=0.6$ and $1.1 \mathrm{~mm} / \mathrm{s}$, Fig. 8 (b) and (c) illustrate that very little tracking were achieved in a small region surrounding the center of the spirals where the frequency components of the input signals have increased to well beyond the bandwidth of the closed-loop system. Nonetheless, Table I shows that the $E_{r m s}$ for the CLV spirals is relatively small since most of the tracking errors were limited only to the center of the resulting spiral scan.

Having analyzed the performance of the closed-loop system in tracking the CLV spirals, we then moved on to investigate the use of spiral scanning in generating AFM images. The spiral scans were setup to produce images with $r_{\text {end }}=6.5 \mu \mathrm{m}$ and number of curves $=512$. However, before performing the spiral scans, the RMS of mapping errors $E_{\text {map }_{R M S}}$ for the CLV 

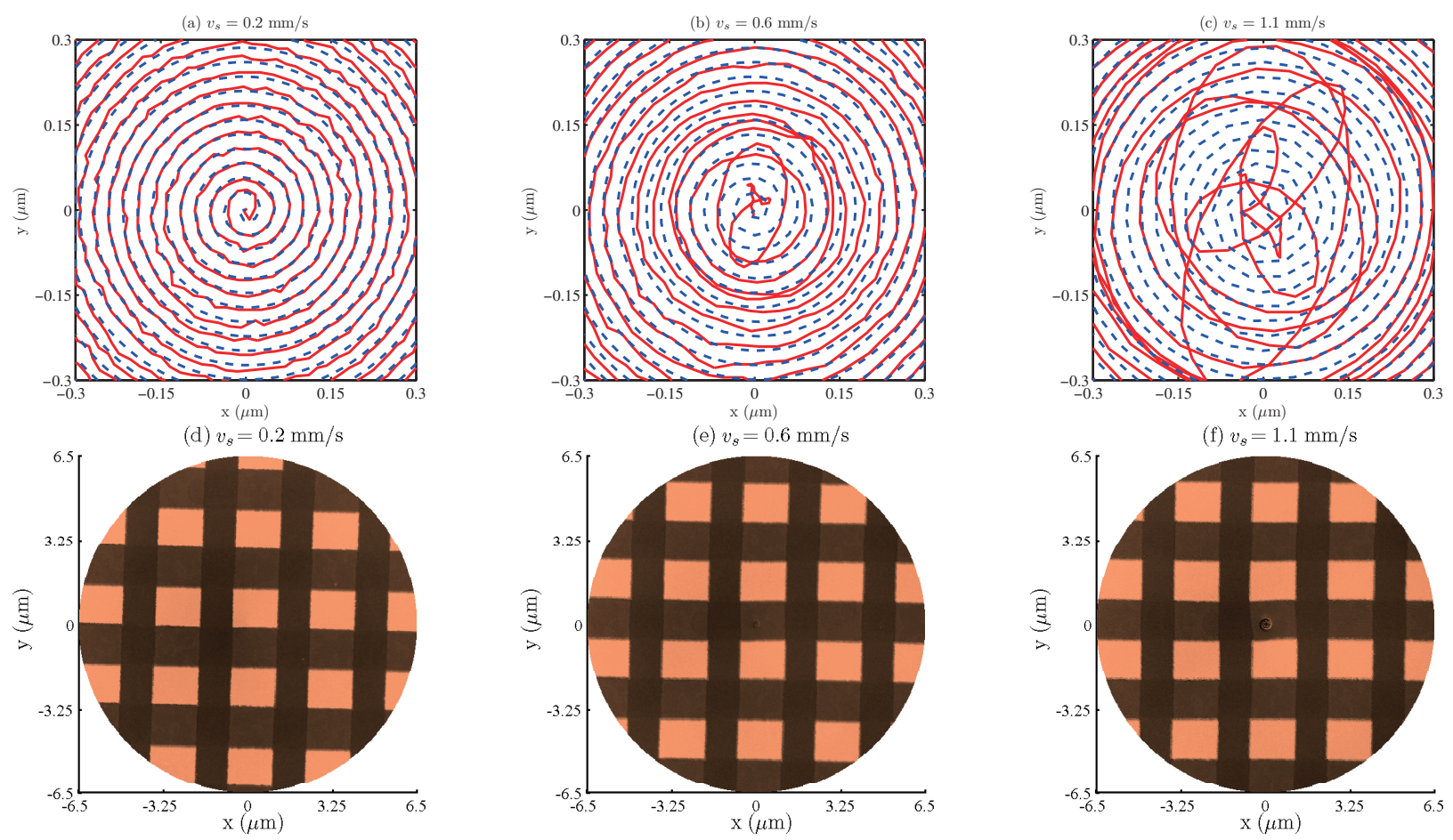

Figure 8. First row: (a) - (c) Tracking trajectories of CLV spirals between $\pm 0.30 \mu \mathrm{m}$ in closed-loop for $v_{s}=0.2,0.6$ and $1.1 \mathrm{~mm} / \mathrm{s}$. Solid line is the achieved response and dashed line is the desired trajectory. Second row: (d) - (f) AFM images of NT-MDT TGQ1 grating scanned in closed-loop using the CLV spiral scanning mode for $v_{s}=0.2,0.6$ and $1.1 \mathrm{~mm} / \mathrm{s}$. The pitch of the spirals was set at $0.0235 \mu \mathrm{m}$ and the number of curve for these AFM images was set to 512 .

spirals scans at different linear velocities are calculated and tabulated in Table I. Note that, different sampling frequencies $f_{\text {samp }}$ were used in order to minimize the computing time for searching the nearest spiral point corresponding the each raster point. Table I shows that the $E_{m a p_{R M S}}$ are very small and less then the pitch of the spiral trajectory and the raster points, i.e., $25.44 \mathrm{~nm}$. Thus, they can be ignored.

A calibration grating NT-MDT TGQ1 with a $20 \mathrm{~nm}$ featureheight and a $3 \mu \mathrm{m}$ period was used as an imaging sample. The AFM was setup to scan the sample in constant-height contact mode using a contact AFM probe with a nominal spring constant of $0.2 \mathrm{~N} / \mathrm{m}$ and resonance frequency of $13 \mathrm{kHz}$. Fig. 8 (d), (e) and (f) illustrate the generated AFM images for $v_{s}=0.2,0.6$ and $1.1 \mathrm{~mm} / \mathrm{s}$. For $v_{s}=0.2 \mathrm{~mm} / \mathrm{s}$, it can be observed that the profile of the calibration grating was well imaged. This is in agreement with the good tracking performance achieved at this scanning speed as illustrated in Fig. 8 (a). However, for higher values of $v_{s}$, Fig. 8 (e) and (f) illustrate that a small hole-like artifact is formed at the center of each image. This is due to the loss of tracking control when the frequency components of the input signals have increased to well beyond the bandwidth of the closed-loop system. The lack of tracking control has also resulted in a slightly skewed AFM image around the center of the spiral for $v_{s}=1.1 \mathrm{~mm} / \mathrm{s}$.

\section{CONCLUSiOnS AND Future Work}

In conclusion, in this paper we demonstrated how a CLV spiral scans can be used to obtain AFM images. The use of CLV mode spiral scanning requires a high-bandwidth controller for accurate tracking of the input signals. Apart from the above mentioned artifacts formed at the center of the CLV spiral, the obtained AFM images are of good qualities.

\section{REFERENCES}

[1] G. Binnig, C. F. Quate, and C. Gerber, "Atomic force microscope," Phys. Rev. Lett., vol. 56, no. 9, pp. 930-933, Mar. 1986.

[2] E. Meyer, H. J. Hug, and R. Bennewitz, Scanning Probe Microscopy. Germany: Springer, 2004

[3] I. A. Mahmood and S. O. R. Moheimani, "Fast spiral-scan atomic force microscopy," Nanotechnology, 2009.

[4] A. N. Labinsky, G. A. J. Reynolds, and J. Halliday, "A disk recording system and a method of controlling the rotation of a turn table in such a disk recording system," WO Patent, vol. 93/13524, July 1993.

[5] B. Bhikkaji, M. Ratnam, A. Fleming, and S. Moheimani, "Highperformance control of piezoelectric tube scanners," Control Systems Technology, IEEE Transactions on, vol. 15, no. 5, pp. 853 - 866, Sept. 2007.

[6] A. J. Fleming and S. O. R. Moheimani, "Sensorless vibration suppression and scan compensation for piezoelectric tube nanopositioners," Control Systems Technology, IEEE Transactions on, vol. 14, no. 1, pp. 33 - 44, Jan. 2006

[7] A. Fleming and K. Leang, "Charge drives for scanning probe microscope positioning stages," Ultramicroscopy, vol. 108, no. 12, pp. $1551-1557$, 2008.

[8] T. McKelvey, H. Akcay, and L. Ljung, "Subspace-based multivariable system identification from frequency response data," Automatic Control, IEEE Transactions on, vol. 41, no. 7, pp. 960 - 979, July 1996.

[9] T. McKelvey, A. Fleming, and S. O. R. Moheimani, "Subspace-based system identification for an acoustic enclosure," Journal of Vibration and Acoustics, vol. 124, no. 3, pp. $414-419,2002$.

[10] S. O. R. Moheimani and A. J. Fleming, Piezoelectric Transducers for Vibration Control and Damping. Germany: Springer, 2006.

[11] J. L. Fanson and T. K. Caughey, "Positive position feedback-control for large space structures," AIAA Journal, vol. 28, no. 4, pp. 717 - 724, April 1990.

[12] A. Lanzon and I. Petersen, "Stability robustness of a feedback interconnection of systems with negative imaginary frequency response," Automatic Control, IEEE Transactions on, vol. 53, no. 4, pp. 10421046, May 2008. 